\title{
Building a new bridge between metabolism, free radicals and longevity
}

\section{Markus Ralser and Hans Lehrach}

\section{Max Planck Institute for Molecular Genetics, 14195 Berlin, Germany}

\author{
Running title: Mitochondrial back-signaling, Afo1 and aging \\ Key words: aging, free radicals, mitochondria, ribosome biogenesis \\ Correspondence: Markus Ralser, Max Planck Institute for Molecular Genetics, Ihnestrasse 73, 14195 Berlin, Germany \\ Received: 09/17/09; accepted: 09/25/09; published on line: 09/26/09 \\ E-mail: ralser@molgen.mpg.de
}

Copyright: (C) 2009 Ralser and Lehrach. This is an open-access article distributed under the terms of the Creative Commons

Attribution License, which permits unrestricted use, distribution, and reproduction in any medium, provided the original author and source are credited

The free radical theory of aging implies that oxidative stress, caused by metabolic activity, is a key factor of the aging process. In the last years, this theory has often been criticized: the mechanistic connections between stress resistance, metabolic activity and oxidative damage, on the one hand, hormesis and longevity on the other, are still elusive. The discovery of a novel genetic factor, AFO1, blows fresh wind into this established theorem. Although afo1s cells lack functional mitochondria, they grow at wild-type rates and live exceptionally long [1]. Responsible is a regulatory crosstalk of mitochondria with the TOR pathway and the transcription factor Sfp1.

Aging is a consequence of metabolic activity, and affects all living organisms. It is believed that unavoidable macromolecular damage, for instance caused by oxidation, is a contributor to the aging process. Indeed, oxidative damage and the concentration of ROS rises with age; many long living mutants confer resistance to oxidative stress [2, 3]. Moreover, calorie restriction or limited caloric intake, causes a reduction in the metabolic turnover, free radical production, and extends lifespan in a variety of organisms [4].

However, the correlation between oxidative stress resistance and aging is not linear. For instance, lifespan extending caloric restriction causes an increase of mitochondrial activity and free radical production in $C$. elegans; lifespan-extension is prevented by anti-oxidant treatments [5]. In yeast, oxidative stress resistance is not a predictor for its lifespan. For instance, mutations in trio- sephosphate isomerase, a central glycolytic enzyme, reduces glycolytic activity and increases oxidative stress resistance, but causes premature aging [6]. A recent genome wide analysis revealed that many genes defective in mitochondrial activity are particularly sensitive to aging [7] Finally, continuous minimal exposures to oxidative conditions seem to be required to maintain the activity of antioxidant-defence systems during life; the principle of hormesis is crucial for natural lifespan [8].

Several attempts have been made to explain these conflicting observations. Blagosklonny, for instance, reminds us that Aging causes damage, not damage causes aging [9]. Following this view, central signaling systems such as the TOR pathway are the causal players of aging; the increase in molecular damage has to be regarded as the consequence rather than the cause of this process.

Indeed, all living organisms are adapted to face a natural amount of free radicals. Therefore, every manipulation of the redox state or metabolic activity targets the natural anti-oxidative machinery. Thus, it is difficult to distinguish between the direct and indirect consequences of a pro- and anti-oxidative exposure in aging experiments. Treatment with hydrogen peroxide, for instance, causes a major and time-dependent rearrangement of the cellular transcriptome and proteome $[10,11]$. This list of oxidant-regulated proteins contains several enzymes from central metabolism; many of them are implicated in pathways with a known role in the aging process. To shed new 
light on these interrelations, Heeren et al. performed a comprehensive analysis that led to the identification of a new factor which positions at the interface of metabolism, mitochondrial activity, free radicals and aging [1]. The authors compared the transcriptome of young and old cells, separated by elutriation centrifugation [12]. Then, deletion mutants of 92 differentially regulated transcripts were tested for resistance against oxidants. Only one gene deletion was resistant to more than two oxidants and showed an extended replicative lifespan in a subsequent micro-dissection experiment: $\triangle Y G R 076 C$, encoding for a mitochondrial ribosomal protein of the large subunit, MRPL25.

The aging phenotype of $\triangle Y G R 076 C$ is remarkable: compared to corresponding wild-type yeast, the strain exhibits a $60 \%$ increase in the median-, and $71 \%$ in the maximum lifespan. Therefore, Heeren et al. named the yet uncharacterized gene Aging factor one (AFO1).

It turns out that $\Delta a f o 1$ cells lack functional mitochondria, a phenotype commonly described as $\rho 0$. The surprising result of this mutant, however, is that $\triangle a f o 1$ yeast has no obvious growth defect on glucose containing media. Usually, $\rho 0$ cells are growing slowly. This result allows a quite remarkable conclusion: at least on glucose media, $\rho 0$ yeast is not decelerating growth because of energetic deficits, the cells are capable to generate sufficient energy by fermenting glucose. But are there other reasons for $\rho 0$ yeast to reduce the growth rate?

Heeren et al. provide evidence that feedback signaling from the mitochondria to factors of ribosome biogenesis slows the growth of $\rho 0$ cells, the Target of Rapamycin (TOR) pathway and the activation of the transcription factor Sfp1 appear to be crucial for this phenotype. Both, the TOR pathway and Sfp1 are known regulators of ribosome biogenesis in response to nutrient limitations and stress response [13-15]; indeed the TOR mediated control of growth rate is an important determinant of lifespan and aging [16].

The rationale for the existence of this signaling system could be the following: Ribosome biogenesis is the most energy consuming cellular process; slowing it down is beneficial when a collapse of the energy status is imminent. A cellular sensing system, termed mitochondrial back signaling, monitors mitochondrial activity and is able to interfere with ribosome biogenesis when required. Cells lacking AFO1 are deficient in this cascade. Therefore, even when the mitochondrial ATP production is zero, ribosome biogenesis continues; $\Delta a f o 1$ cells grow at normal speed. Since this strain lacks a functional respiratory chain, it produces fewer free radicals and thus suffers less from macromolecular damage.

But why did yeast not loose mitochondrial back signaling during evolution? First, yeast is not evolutionarily selected for longevity. Because they are larger and divide at slower rates, old cells may have a selective disadvantage when competing with younger cells. And, although oxidized molecules are tightly kept with the mother during cell division, old mothers have a higher risk to transmit damaged macromolecules to their daughters [17]. Second, the high calorie supply in the early growth phase ( $2 \%$ glucose) is a quite artificial condition; in the natural environment, competition for nourishments is one of the driving forces of evolution [18]. Therefore, communication between processes that produce and consume energy is highly advantageous and probably essential to survive in a natural environment.

\section{BOX1 afo1 phenotypes}

- Differentially regulated between old and young yeast cells

- Deletion mutant: resistant to diamide, tert-butyl hydroperoxide and hydrogen peroxide

- $50 \%$ decrease in ROS formation

- respiratory deficient, pet- phenotype, does not grow on nonfermentable carbon source, lacks mitochondrial DNA

- normal growth rate on glucose media

- $60 \%$ increase in median replicative lifespan

- $71 \%$ increase in maximum replicative lifespan 
The discovery of AFO1 establishes a new connection between mitochondria, ribosome biogenesis, free radicals and aging. Future studies have to deepen the knowledge about the activity and control of metabolic pathways in this interesting mutant without mitochondrial respiration; further investigations will provide fruitful new insights into the role of free radicals in the aging process. Indirectly, however, the study of Heeren et al. prompts for a careful reexamination of many conclusions drawn from the use of oxidants, anti-oxidants, calorie restrictions and other metabolic perturbations when studying aging: lifespanextending phenotypes could often be a result from the activation of yet unknown signaling systems, and not a direct biochemical consequence of the studied treatment.

\section{ACKNOWLEDGEMENTS}

We are grateful to our lab-members for critical reading of the manuscript and to the Max Planck Society for funding.

\section{CONFLICT OF INTERESTS STATEMENT}

The authors declare no conflict of interests.

\section{REFERENCES}

1. Heeren $G$, et al. The mitochondrial ribosomal protein of the large subunit, Afo1p, determines cellular longevity through mitochondrial back-signaling via TOR1. Aging. 2009; 1: 622-636.

2. Johnson FB, et al. Molecular biology of aging. Cell. 1999; 96:291-302.

3. Finkel $\mathrm{T}$, and Holbrook NJ. Oxidants, oxidative stress and the biology of ageing. Nature. 2000; 408:239-247.

4. Masoro EJ. Overview of caloric restriction and ageing. Mech Ageing Dev. 2005; 126:913-922.

5. Schulz TJ, et al. Glucose restriction extends Caenorhabditis elegans life span by inducing mitochondrial respiration and increasing oxidative stress. Cell Metab. 2007; 6:280-293.

6. Ralser $\mathrm{M}$, et al. Dynamic rerouting of the carbohydrate flux is key to counteracting oxidative stress. J Biol. 2007; 6:10.

7. Merz S, and Westermann B. Genome-wide deletion mutant analysis reveals genes required for respiratory growth, mitochondrial genome maintenance and mitochondrial protein synthesis in Saccharomyces cerevisiae. Genome biology. 2009; 10:R95.

8. Rattan SI. Hormesis in aging. Ageing Res Rev. 2008; 7:63-78.

9. Blagosklonny MV. Aging: ROS or TOR. Cell Cycle. 2008; 7:33443354.

10. Godon C, et al. The $\mathrm{H} 2 \mathrm{O} 2$ stimulon in Saccharomyces cerevisiae. J Biol Chem.1998; 273:22480-22489.

11. Chechik $G$, et al. Activity motifs reveal principles of timing in transcriptional control of the yeast metabolic network. Nat Biotechnol. 2008; 26:1251-1259.
12. Laun $P$, et al. Aged mother cells of Saccharomyces cerevisiae show markers of oxidative stress and apoptosis. Molecular microbiology. 2001; 39:1166-1173.

13. Hall MN. (2008) mTOR-what does it do? Transplantation proceedings $40, \mathrm{~S} 5-8$.

14. Steinberg, GR, and Kemp BE. AMPK in Health and Disease. Physiological reviews. 2009; 89:1025-1078.

15. Singh J, and Tyers M. A Rab escort protein integrates the secretion system with TOR signaling and ribosome biogenesis. Genes Dev. 2009; 23:1944-1958.

16. Blagosklonny $M$, Hall $M$ : Growth and aging: a common molecular mechanism. Aging. 2009; 1:357-362.

17. Aguilaniu $\mathrm{H}$, et al. Asymmetric inheritance of oxidatively damaged proteins during cytokinesis. Science. 2003; 299:17511753.

18. McNab BK. Food-Habits, Energetics, and the Population Biology of Mammals. American Naturalist. 1980; 116;106-124. 